\title{
TEXTURE AND THERMAL EXPANSION ANOMALIES OF B19'-MARTENSITE IN TENSILE DEFORMED TiNi SHAPE MEMORY ALLOY
}

\author{
V.M. GUNDYREV, V.I. ZEL'DOVICH and G.A. SOBYANINA \\ Institute of Metal Physics, Russian Academy of Sciences, \\ S. Kovalevskaya Str., 18, 620219 Ekaterinburg, Russia
}

(Received in final form 28 September 1997)

The dilatometric studies of Ti-50.5 at.\% Ni alloy have shown that the thermal expansion coefficient (TEC) of B19'-martensite varies considerably for the solution-treated and tensile-strained samples. In the undeformed state the TEC has the positive value $\alpha=9.7 \cdot 10^{-6} \mathrm{~K}^{-1}$. After tensile straining the TEC decreases sharply and takes on a negative value (down to $-10.8 \cdot 10^{-6} \mathrm{~K}^{-1}$ ). The reasons for the variation of the TEC of the B19'-phase of the solution-treated and deformed samples are discussed. The tensile straining was found to create in the samples an extremely sharp texture of the phase transformation, with the texture axis parallel to the strain direction. The TEC of monoclinic B19'-lattice is different in various crystallographic directions and assumes a negative value in the $\left[\begin{array}{lll}0 & 0 & 1\end{array}\right]_{\mathrm{B} 19^{\prime}}$ direction.

Keywords: B19'-martensite; Thermal expansion coefficient; Thermal expansion anisotropy; Tensile straining; Texture of martensite

\section{INTRODUCTION}

The thermal expansion of shape memory alloys is significant for their practical applications. It is expected that the texture of B19'-martensite in titanium nickelide will affect the thermal expansion coefficient (Zel'dovich et al., 1996). However, the thermal expansion of B19'martensite of titanium nickelide has not yet been studied. This paper has been the first to deal with the relation between the thermal expansion 
and texture of B19'-martensite of titanium nickelide tensile-strained samples.

\section{MATERIALS AND METHODS}

The material used for this study was in the form of commercially smelted $\mathrm{Ti}_{49,5} \mathrm{Ni}_{50,5}$ slabs. The sheets were produced by hot rolling at a temperature not exceeding $1273 \mathrm{~K}$, annealed, rolled at $773 \mathrm{~K}$, and etched. Samples with dimensions of $125 \times 10 \times 2.2$ and $125 \times 10 \times 1.1 \mathrm{~mm}$ were cut out for the tensile straining. Prior to the straining the samples were quenched from $1073 \mathrm{~K}$ in water. After quenching the samples had a single-component B2-phase texture $\left\{\begin{array}{llll}1 & 1 & 0\end{array}\right\}\left\langle\begin{array}{llll}1 & 1 & 0\end{array}\right)$ (Zel'dovich et al., 1996). The samples were tensile-strained at room temperature (in the state $\mathrm{B} 2+\mathrm{B} 19^{\prime}$ ) at a rate of $1.2 \times 10^{-4} \mathrm{~s}^{-1}$. The degree of residual (after unloading of the samples) plastic strain varied from $0.7 \%$ to $16 \%$. The samples were tensile-loaded in the direction of the previous rolling.

Samples for dilatometric studies were cut from the tension part of the strained samples in the direction of tensile strain. Samples $10 \times 10 \times 1.1 \mathrm{~mm}$ in size for X-ray studies were cut from the surface parallel to the rolling plane (RD-TD surface). In addition, samples $10 \times 10 \times 1.5 \mathrm{~mm}$ in size were completed from the cutting strips with dimensions $10 \times 1.1 \times 1.5 \mathrm{~mm}$ for $\mathrm{X}$-ray studies from surface perpendicular to the tensile strain direction (ND-TD surface). The samples were prepared in the temperature interval from 283 to $295 \mathrm{~K}$, because the finish temperature of the reverse transformation $\mathrm{B} 19^{\prime} \rightarrow \mathrm{B} 2$ was higher than $295 \mathrm{~K}$ and the start temperature of the direct transformation $\mathrm{B} 2 \rightarrow \mathrm{B} 19^{\prime}$ was lower than $283 \mathrm{~K}$.

The X-ray diffraction study was performed on a DRON-1 diffractometer with $\mathrm{Cu}-\mathrm{K}_{\alpha}$ radiation. The $\mathrm{X}$-ray diffraction patterns were taken during cooling of the specimens from room temperature to $93 \mathrm{~K}$ and during subsequent heating to room temperature. The dilatometric studies were carried out on a Chevenard dilatometer equipped with a furnace and a cryostat. For the dilatometric studies the samples were initially cooled to $77 \mathrm{~K}$ for completion of the martensitic transformation and then heated to $523 \mathrm{~K}$. The absolute values of the linear TEC at temperatures from 173 to $77 \mathrm{~K}$ were measured with a rather large error 
of $1 \cdot 10^{-6} \mathrm{~K}^{-1}$, because of uncertain values of the linear TEC of the reference standard (pyros), which were obtained by extrapolation to low temperatures. The measurement accuracy of the relative change in the thermal expansion coefficient in different states was an orderof-magnitude higher, i.e., $0.05 \cdot 10^{-6} \mathrm{~K}^{-1}$.

The samples prior to straining contained to B2-phase and a small amount of the B19'-martensite. Figure 1 shows a tensile stress-strain diagram on which the maximum strains for the samples subjected to the dilatometric study are indicated. The diagram consists of three segments. The first segment of the diagram, at strains up to $0.2 \%$, is elastic. The second segment, at strains up to $\sim 5 \%$, corresponds mainly to the transformation $\mathrm{B} 2 \rightarrow \mathrm{B} 19^{\prime}$. The third segment, which extends to the point of fracture, reflects the strain of the B19'-martensitic structure. Subsequently we used the samples, which correspond to the end of the second segment $(4.7 \%)$ and to the beginning, middle, and end of the third segment $(6.7 \%, 10 \%, 12 \%$ and $16 \%)$.

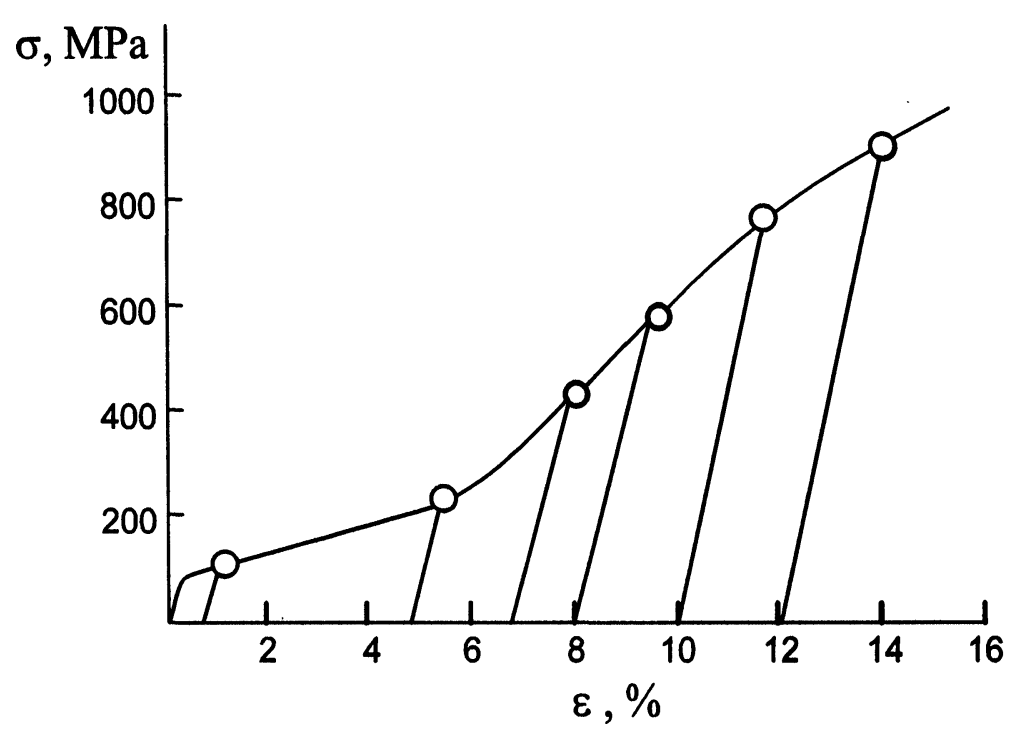

FIGURE 1 Stress-strain diagram of the Ti-50.5 at.\% Ni alloy. 


\section{RESULTS}

The earlier dilatometric studies of the Ti-50.5 at.\% Ni alloy (Zel'dovich et al., 1996) showed that the thermal expansion coefficients of the B19'phase vary considerably in the temperature interval from 173 to $77 \mathrm{~K}$ (i.e. well below the finish of the martensitic transformations B2 $\rightarrow$ B19' and the start of the reverse transition $\mathrm{B} 19^{\prime} \rightarrow \mathrm{B} 2$ ) for the solution-treated and tensile-strained samples. To illustrate this variability, Fig. 2 shows the dilatometric curves of the martensitic transformations recorded by the standard method (normal recording), with the elongation of the sample plotted along the vertical axis. The numbers at the curves indicate the sequence of the experiments: cooling $(0-1)$ and heating (1-2). From the curves it is seen that after quenching the thermal expansion coefficients is positive, while after tensile straining the TEC of the B19' is negative. The heating-cooling-heating cycle performed over the temperature interval from 173 to $77 \mathrm{~K}$ confirmed the reversibility of the dilatometric curve.

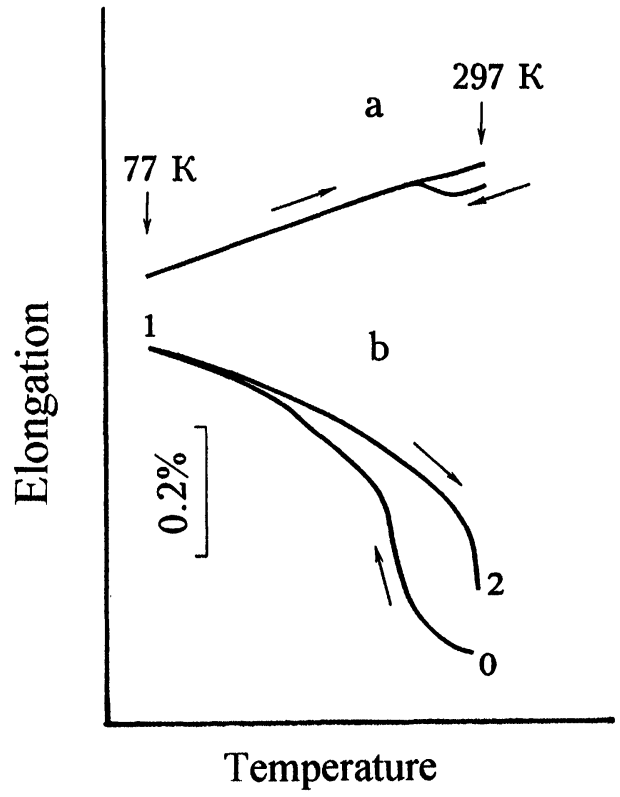

FIGURE 2 Dilatometric curves of the martensitic transformations after quenching (a) and after tensile straining to $6.7 \%$ (b). Normal recording. 
Let us discuss the variation of the linear TEC of titanium nickelide in the $\mathrm{B} 19^{\prime}$ state as a function of the degree of plastic deformation. In the undeformed state the TEC has the value $\alpha=9.7 \cdot 10^{-6} \mathrm{~K}^{-1}$. After tensile-straining the TEC decreases sharply and takes on negative values. The minimum value of the TEC $\left(-10.8 \cdot 10^{-6} \mathrm{~K}^{-1}\right)$ is reached at the strain of $12 \%$. This result may be explained as follows. The B19'martensite formed during straining is textured: out of all the crystallographically equivalent orientations of martensite, those are realized that give the maximum contribution to the strain. According to the orientation relationship of the B2- and B19'-phases reported by Khachin et al. (1992), the maximum contribution to the strain (about $8 \%$ ) is due to the orientation which is aligned in the $\left[\begin{array}{lll}0 & 1 & 1\end{array}\right]_{\mathrm{B} 2}$ direction with the $\left[\begin{array}{lll}0 & 1\end{array}\right]_{\mathrm{B} 19^{\prime}}$ axis, having a period $4.622 \AA$ (Shamray et al., 1995).

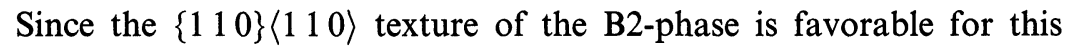
version of the orientation relationship, one may suppose that tensile straining of the samples creates an extremely sharp texture by the phase transformation, with the texture axis parallel to the rolling direction.

Thus, comparison of the expected texture of the B19' phase and measured TEC reveals two factors. First, the linear TEC of the monoclinic B19' lattice is different in different crystallographic directions, and, in particular, it takes on a negative value in the $\left[\begin{array}{lll}0 & 0 & 1\end{array}\right]_{\mathrm{B} 19^{\prime}}$ direction, while the volume expansion coefficient is positive. Titanium nickelide is not unique in having this sort of behavior of the thermal expansion. There are a few other crystals which have a negative value of individual thermal expansion coefficients (Nye, 1960). Second, the value of the TEC may be an indication of the degree of perfection of the $\left[\begin{array}{lll}0 & 0 & 1\end{array}\right]_{\mathrm{B} 19^{\prime}}$ texture.

To verify the presence of the texture in the martensite, the samples underwent an X-ray examination. Diffractograms were taken from the surface normal to the tensile strain direction (ND-TD surface) and from a surface parallel to the rolling plane (RD-TD surface). In the undeformed state the relative intensities of the $(002)$ and $(020)$ lines of B19'-martensite were nearly equal in the diffractograms taken from the ND-TD and RD-TD surfaces. This is characteristic of a slightly textured material. Figure 3 (curves 1 and 2) shows portions of the diffractograms of the strained sample. It is seen that the intensity of the $\left(\begin{array}{lll}0 & 0\end{array}\right)$ line of $\mathrm{B} 19^{\prime}$-martensite is high, while the $\left(\begin{array}{ll}0 & 2\end{array}\right)$ line is absent in the diffractogram taken from the ND-TD surface. On the contrary, 

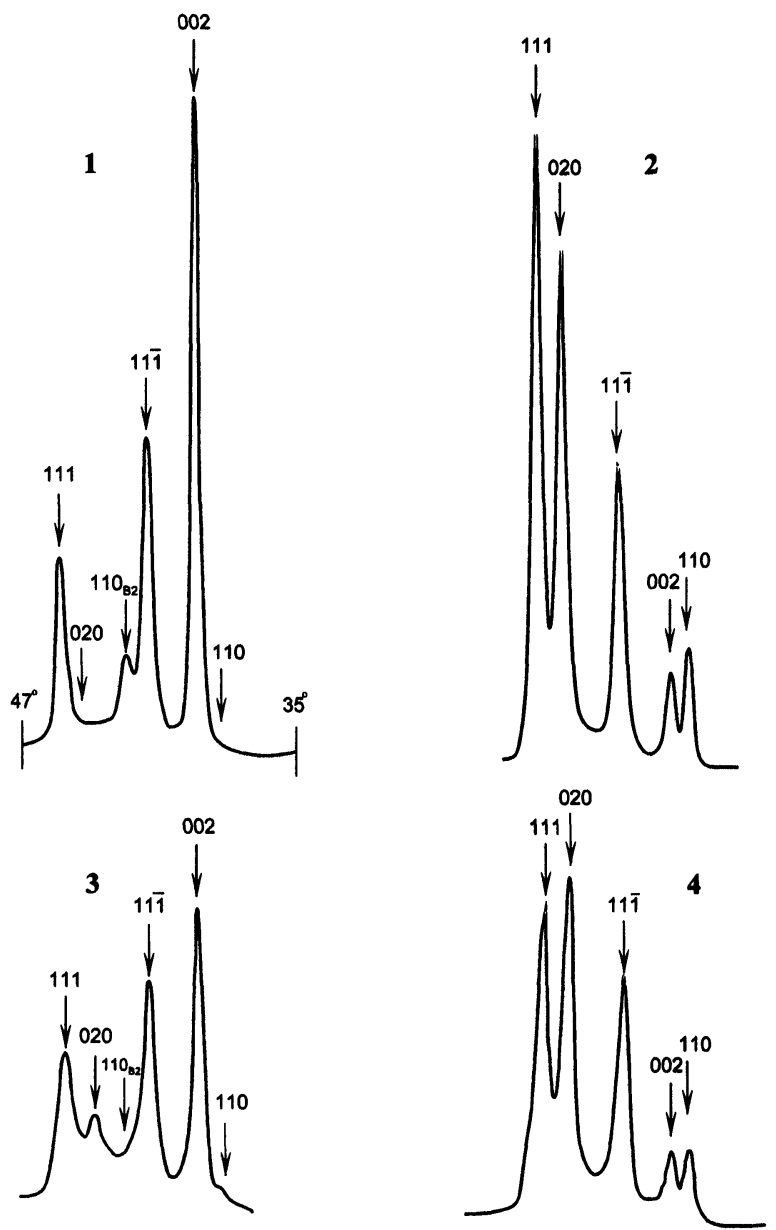

FIGURE 3 Fragments of X-ray diffractograms taken after tensile straining to $10 \%$ from ND-TD (1) and RD-TD (2) surfaces and after tensile straining to $10 \%$ followed by the $\mathrm{B} 2 \rightarrow \mathrm{B} 19^{\prime} \rightarrow \mathrm{B} 2$ transformations from ND-TD (3) and RD-TD (4) surfaces.

in the diffractogram taken from the RD-TD surface the intensity of the $\left(\begin{array}{ll}0 & 2\end{array}\right)$ line is much higher than that of $\left(\begin{array}{ll}0 & 02\end{array}\right)$. Therefore, the $\left(\begin{array}{ll}0 & 02\end{array}\right)$ planes lie mostly perpendicular to the direction of tensile straining. The finer details of the orientation of the crystallites can be seen from the pole figure in Fig. 4. If the monoclinic distortion of the lattice is neglected, the $\left[\begin{array}{ll}0 & 0\end{array}\right]$ direction of the $\mathrm{B} 19^{\prime}$ phase is assumed to be perpendicular to 


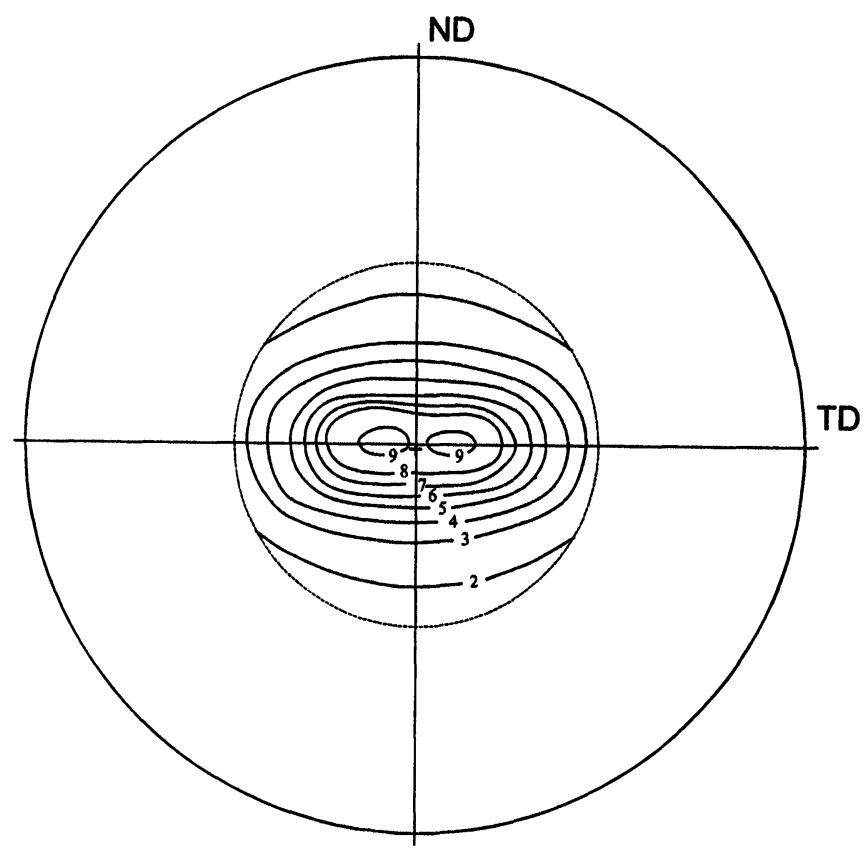

FIGURE 4 Central part of the pole figure for $(002)$ B $19^{\prime}$-martensite after a tensile straining to $10 \%$ (TD stands for the transverse direction; the numbers denote the relative intensity).

the (002) plane, and splitting of the texture maximum observed in the pole figure is disregarded, it may be inferred that the martensite texture with the [ $\left[\begin{array}{ll}0 & 1\end{array}\right]$ axis is located along the rolling direction (the direction of tensile straining). During the subsequent heat treatment, which is accompanied by the transformations $\mathrm{B} 19^{\prime} \rightarrow \mathrm{B} 2 \rightarrow \mathrm{B} 19^{\prime}$, the texture deteriorates in accordance with the prediction as may be seen from the relative intensities of the $\left(\begin{array}{ll}0 & 02\end{array}\right)$ and $(020)$ lines in Fig. 3 (curves 3 and 4 ).

To obtain additional information about thermal expansion of the B19'-phase along different crystallographic axes, the B19'-phase interplanar spacings vs temperature were examined by an X-ray method. Figure 5 shows the temperature dependences of the interplanar spacings $\mathrm{d}_{002}, \mathrm{~d}_{11 \overline{1}}, \mathrm{~d}_{020}$ and $\mathrm{d}_{111}$. The numbers at the curves and in the text below indicate the treatments and the irradiation surface of the samples: (1) after tensile straining (first B2 $\rightarrow$ B19' transformation), ND-TD surface; (2) after tensile straining, first B2 $\rightarrow$ B19' transformation, RD-TD 


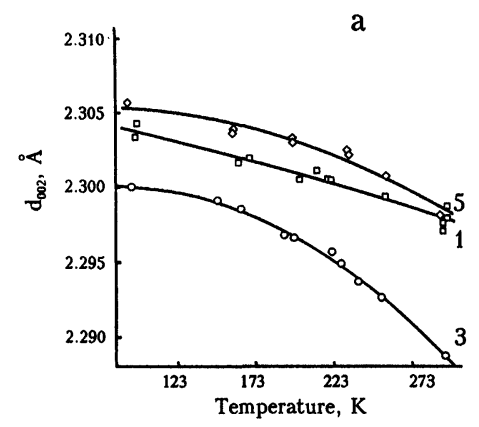

b
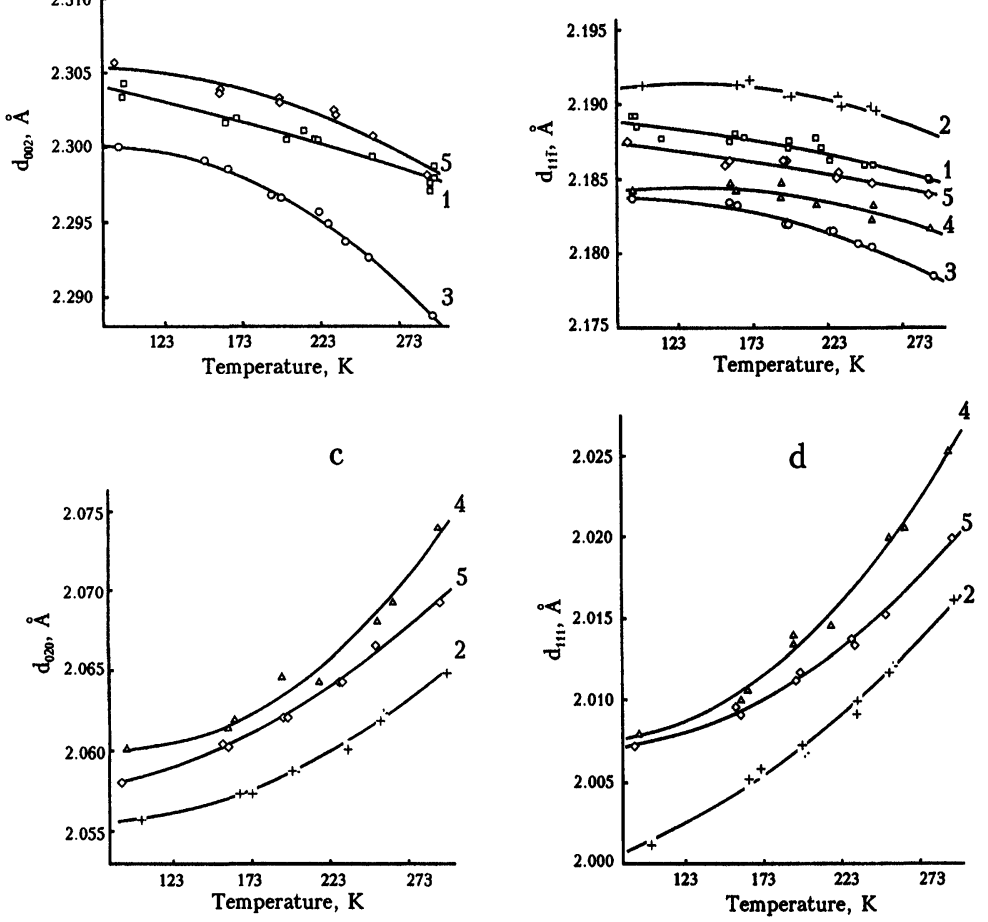

FIGURE 5 Temperature dependences of interplanar spacings $d_{002}(a), d_{11 \overline{1}}(b), d_{020}(c)$, $\mathrm{d}_{111}$ (d) for strong lines.

surface; (3) after tensile straining and followed by $\mathrm{B} 2 \rightarrow \mathrm{B} 19^{\prime} \rightarrow$ $\mathrm{B} 2 \rightarrow \mathrm{B} 19^{\prime}$ transformations (second $\mathrm{B} 2 \rightarrow \mathrm{B} 19^{\prime}$ transformation), NDTD surface; (4) second $\mathrm{B} 2 \rightarrow \mathrm{B} 19^{\prime}$ transformation, RD-TD surface; (5) after quenching (unstrained sample), ND-TD surface.

The temperature dependences of $B 19^{\prime}$ interplanar spacings $\mathrm{d}_{\mathrm{hk} 1}$ (Fig. 5) show that the interplanar spacings measured at room temperature for different sections of one and the same sample and for samples undergoing different treatments (quenching, deformation, deformation followed by the $\mathrm{B} 19^{\prime} \rightarrow \mathrm{B} 2 \rightarrow \mathrm{B} 19^{\prime}$ transformations) differ considerably. This fact suggests that large residual stresses remain in crystallites after the martensitic transition $\mathrm{B} 2 \rightarrow \mathrm{B} 19^{\prime}$. The maximum internal stresses calculated using the Young's modulus of $6800 \mathrm{~kg} / \mathrm{mm}^{2}$ reported by Kornilov et al. (1977) are equal to $\sim 25 \mathrm{~kg} / \mathrm{mm}^{2}$. 
Note that the temperature dependences of the interplanar spacings for strong lines differ from those for weak lines (see Figs. 5 and 6). The preferable orientation of $\mathrm{B}^{\prime} \mathrm{9}^{\prime}$ crystallites is characterized by the direction of the [0 02 2] axis aligned with the RD (Fig. 4). Strong 002 (1), 111 (2) and 020 (2) lines also correspond to the preferable orientation
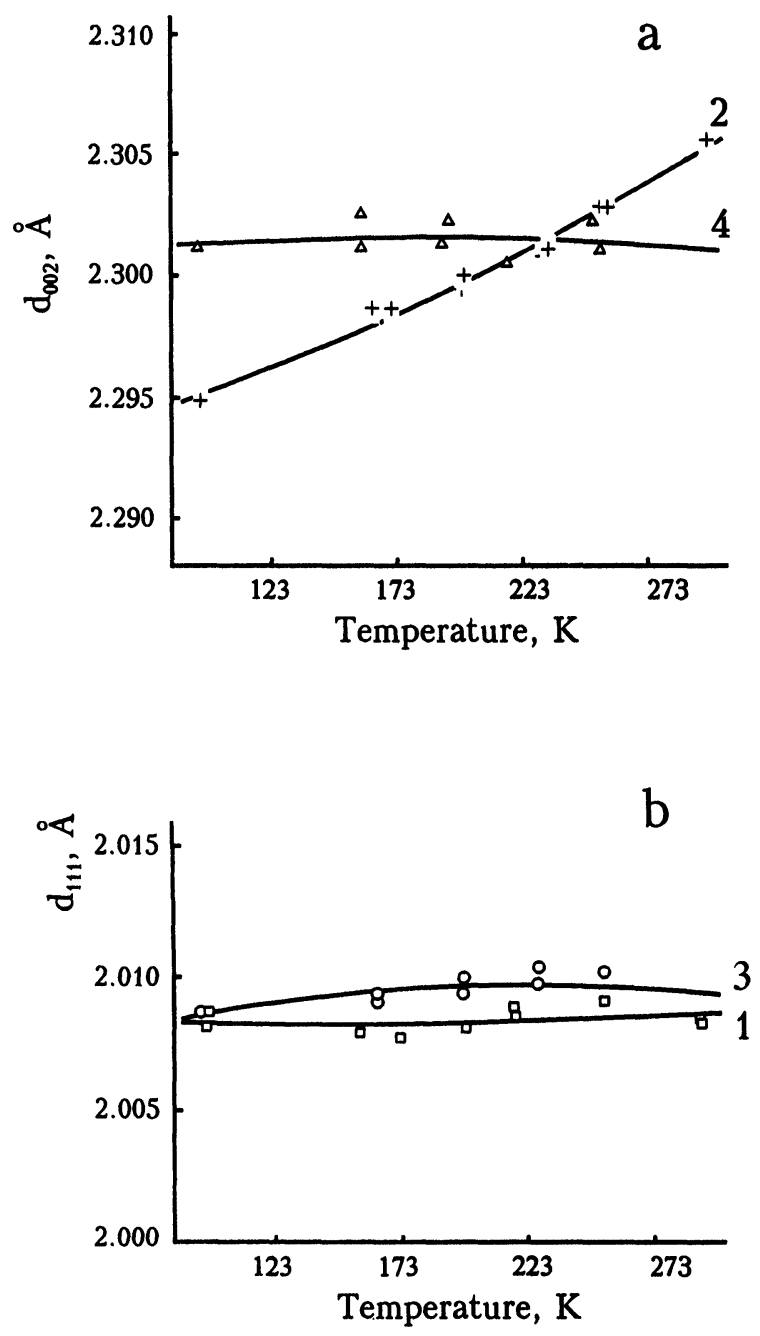

FIGURE 6 Temperature dependences of interplanar spacings $d_{002}(a), d_{111}$ (b) for weak lines. 
TABLE I Thermal expansion coefficients of predominant orientation crystallites calculated from curves on Fig. 5

\begin{tabular}{lc}
\hline Indexes of planes and irradiation surface & $\alpha \cdot 10^{6}\left(\mathrm{~K}^{-1}\right)$ \\
\hline $002(1)$ & -11 \\
$020(2)$ & 12 \\
$111(2)$ & 26 \\
\hline
\end{tabular}

(see Fig. 3). The curves 002 (1), 002 (3), 111 (2), 111 (4), 020 (2) and 020 (4) of the dominant crystallites clearly show that these crystallites

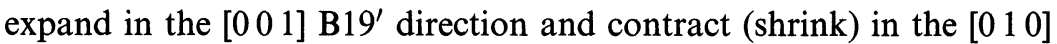
and [ $\left[\begin{array}{lll}1 & 1 & 1\end{array}\right]$ directions with decreasing temperature. The TEC of the tensile-strained sample calculated for the temperature interval from 173 to $93 \mathrm{~K}$ showed a thermal expansion anisotropy given in Table $\mathrm{I}$.

The TEC calculated from the results of dilatometric measurements in the $\left[\begin{array}{lll}0 & 0 & 1\end{array}\right] \mathrm{B} 19^{\prime}$ direction $\left(-10.8 \cdot 10^{-6} \mathrm{~K}^{-1}\right)$ nearly coincides with the TEC calculated using the X-ray findings for the 002 (1) line $\left(-11 \cdot 10^{-6} \mathrm{~K}^{-1}\right)$. However, the TEC determined from the dilatometric measurements must be much smaller if one considers the texture scattering and constrained conditions of the internal crystallites. The X-ray examinations provided information about crystallites located only in the surface layer of sample. For this reason the TEC calculated from the $\mathrm{X}$-ray findings reflects not only the true thermal expansion but also the effect of the residual stresses which change with temperature.

The crystallites, which deviate most from the preferable orientation are characterized by weak 111 (1) and 002 (2) lines (see Fig. 3, curves 1 and 2). Stresses arising between dominant and weak crystallites as a result of the thermal expansion anisotropy lead to a considerable deformation of the latter. Therefore the behavior of the curves 002 (2), 002 (4), 111 (1) and 111 (3) in Fig. 6 is determined by the deformation of the crystallites rather than their thermal expansion.

Thus, the negative TEC determined during the dilatometric measurements is due both to the true thermal expansion and the residual stresses.

\section{References}

Khachin, V.N., Pushin, V.G. and Kondratjev, V.V. (1992). Titanium Nickelide. Structure and Properties (in Russian), pp. 30-76.

Kornilov, I.I., Belousov, O.K. and Kachur, E.V. (1977). Titanium Nickelide and Other Shape Memory Alloys (in Russian), p. 126. 
Nye, J.F. (1960). Physical Properties of Crystals (in Russian), pp. 130-132.

Shamray, V.F., Rubina, E.B., Zhurin, S.A. and Proskurin, V.B. (1995). Structural changes in NiTiNOL alloy during thermomechanical cycling. Fizika Metallov $i$ Metallovedenie, 80, 78-85.

Zel'dovich, V.I., Sobyanina, G.A. and Rinkevich, O.S. (1996). Influence of prestrain on the shape memory effect and martensite structure in titanium nickelide: I. Dilatometric effects of martensitic transformations. The Physics of Metals and Metallography, 81, 305-312. 